Proyecciones Journal of Mathematics

Vol. 41, $\mathrm{N}^{o}$ 1, pp. 275-300, February 2022.

Universidad Católica del Norte

Antofagasta - Chile

\title{
New inequalities for strongly exponentially generalized functions with applications
}

\author{
Artion Kashuri \\ University Ismail Qemali, Albania \\ and \\ Rozana Liko \\ University Ismail Qemali, Albania \\ Received : July 2019. Accepted : December 2020
}

\begin{abstract}
The aim of this paper is to introduce a new class of functions called strongly exponentially generalized $\left(m, \nu_{1}, \nu_{2}, g_{1}, g_{2}\right)$. Some new integral inequalities of trapezium-type for strongly exponentially generalized $\left(m, \nu_{1}, \nu_{2}, g_{1}, g_{2}\right)$ functions with modulus c via Riemann-Liouville fractional integral are established. Also, some new estimates with respect to trapezium-type integral inequalities for strongly exponentially generalized $\left(m, \nu_{1}, \nu_{2}, g_{1}, g_{2}\right)$ functions with modulus $c$ via general fractional integrals are obtained. We show that the strongly exponentially generalized $\left(m, \nu_{1}, \nu_{2}, g_{1}, g_{2}\right)$ functions with modulus $c$ includes several other classes of functions. At the end, some new error estimates for trapezoidal quadrature formula are provided as well. This results may stimulate further research in different areas of pure and applied sciences.
\end{abstract}

2010 Mathematics Subject Classification: Primary: 26D0\%; Secondary: 26A51, 26A33, 26D10, 26D15, 90C23.

Keywords: Trapezium-type integral inequalities, preinvexity, exponential convex function, general fractional integrals. 


\section{Introduction}

The class of convex functions is well known in the literature and is usually defined in the following way:

Definition 1.1. Let $I$ be an interval in $\mathbf{R}$. A function $\varsigma: I \rightarrow \mathbf{R}$, is said to be convex on $I$ if the inequality

$$
\varsigma\left(t \imath_{1}+(1-t) \imath_{2}\right) \leq t \varsigma\left(\imath_{1}\right)+(1-t) \varsigma\left(\imath_{2}\right)
$$

holds for all $\imath_{1}, \imath_{2} \in I$ and $t \in[0,1]$. Also, we say that $\varsigma$ is concave, if the inequality in (1.1) holds in the reverse direction.

The following inequality, named Hermite-Hadamard inequality, is one of the most famous inequalities in the literature for convex functions.

Theorem 1.2. Let $\varsigma: I \subseteq \mathbf{R} \rightarrow \mathbf{R}$ be a convex function and $\imath_{1}, \imath_{2} \in I$ with $\imath_{1}<\imath_{2}$. Then the following inequality holds:

$$
\varsigma\left(\frac{\imath_{1}+\imath_{2}}{2}\right) \leq \frac{1}{\imath_{2}-\imath_{1}} \int_{\imath_{1}}^{\imath_{2}} \varsigma(x) d x \leq \frac{\varsigma\left(\imath_{1}\right)+\varsigma\left(\imath_{2}\right)}{2} .
$$

This inequality (1.2) is also known as trapezium inequality.

The trapezium inequality has remained an area of great interest due to its wide applications in the field of mathematical analysis. Authors of recent decades have studied (1.2) in the premises of newly invented definitions due to motivation of convex function. Interested readers see the references [4]-[12],[15]-[21],[29, 30, 36, 38, 39].

Now, let us recall the following definitions.

Definition 1.3. [16] A function $\varsigma: I \subseteq \mathbf{R} \longrightarrow \mathbf{R}$ is called strongly convex with modulus $c \in \mathbf{R}^{+}$, if

$$
\varsigma(t x+(1-t) y) \leq t \varsigma(x)+(1-t) \varsigma(y)-c t(1-t)(x-y)^{2}
$$

holds for every $x, y \in I$ and $t \in[0,1]$.

Strongly convex functions have been introduced by Polyak, see [16] and references therein. Since strong convexity is a strengthening of the notion of convexity, some properties of strongly convex functions are just stronger versions of known properties of convex functions. Strongly convex functions have been used for proving the convergence of a gradient type algorithm for minimizing a function. They play an important role in optimization theory and mathematical economics. 
Definition 1.4. $[16,17]$ A function $\varsigma: I \subseteq \mathbf{R} \longrightarrow \mathbf{R}$ is called strongly $m$-convex with $m \in[0,1]$ and modulus $c \in \mathbf{R}^{+}$, if

$$
\varsigma(t x+m(1-t) y) \leq t \varsigma(x)+m(1-t) \varsigma(y)-c m t(1-t)(y-x)^{2}
$$

holds for every $x, y \in I$ and $t \in[0,1]$.

Definition 1.5. [29] A function: $\varsigma: I \subseteq \mathbf{R} \rightarrow \mathbf{R}$ is said to be $m-M T$ convex, if $\varsigma$ is positive and $\forall x, y \in I$, and $t \in(0,1)$, among $m \in(0,1]$, satisfies the following inequality

$$
\varsigma(t x+m(1-t) y) \leq \frac{\sqrt{t}}{2 \sqrt{1-t}} \varsigma(x)+\frac{m \sqrt{1-t}}{2 \sqrt{t}} \varsigma(y) .
$$

Definition 1.6. [3] $A$ set $O \subseteq \mathbf{R}^{n}$ is said to be invex respecting the mapping $\tau: O \times O \rightarrow \mathbf{R}^{n}$, if $x+t \tau(y, x) \in O$ for every $x, y \in O$ and $t \in[0,1]$.

Definition 1.7. [20] Let $g:[0,1] \rightarrow \mathbf{R}$ be a nonnegative function and $g \neq 0$. The function $\varsigma$ on the invex set $O$ is said to be $g$-preinvex with respect to $\tau$, if

$$
\varsigma(x+t \tau(y, x)) \leq g(1-t) \varsigma(x)+g(t) \varsigma(y)
$$

for each $x, y \in O$ and $t \in[0,1]$ where $\varsigma(\cdot)>0$.

Definition 1.8. [11] A set $O \subseteq \mathbf{R}^{n}$ is named as $m$-invex with respect to the mapping $\tau: O \times O \rightarrow \mathbf{R}^{n}$ for some fixed $m \in(0,1]$, if $m x+t \tau(y, m x) \in O$ holds for each $x, y \in O$ and any $t \in[0,1]$.

Remark 1.9. Taking $m=1$ in definition 1.8, the mapping $\tau(y, m x)$ reduce to $\tau(y, x)$, and then we get definition 1.6.

Definition 1.10. [32] Let $O \subseteq \mathbf{R}$ be $m$-invex set respecting the mapping $\tau: O \times O \rightarrow \mathbf{R}$ and $g_{1}, g_{2}:[0,1] \rightarrow[0,+\infty)$. A function $\varsigma: O \rightarrow \mathbf{R}$ is said to be generalized $\left(m, g_{1}, g_{2}\right)$-preinvex, if

$$
\varsigma(m x+t \tau(y, m x)) \leq m g_{1}(t) \varsigma(x)+g_{2}(t) \varsigma(y)
$$

is valid for all $x, y \in O$ and $t \in[0,1]$, for some fixed $m \in(0,1]$. 
Definition 1.11. [18] Let $\varsigma \in L\left[\imath_{1}, \imath_{2}\right]$. The Riemann-Liouville integrals $J_{\imath_{1}+}^{\alpha} \varsigma$ and $J_{\imath_{2}-}^{\alpha} \varsigma$ of order $\alpha>0$ with $\imath_{1} \geq 0$ are defined by

$$
J_{\imath_{1}+}^{\alpha} \varsigma(x)=\frac{1}{\Gamma(\alpha)} \int_{\imath_{1}}^{x}(x-t)^{\alpha-1} \varsigma(t) d t, \quad x>\imath_{1}
$$

and

$$
J_{2_{2}-}^{\alpha} \varsigma(x)=\frac{1}{\Gamma(\alpha)} \int_{x}^{\imath_{2}}(t-x)^{\alpha-1} \varsigma(t) d t, \quad \imath_{2}>x,
$$

where $\Gamma(\alpha)=\int_{0}^{+\infty} e^{-u} u^{\alpha-1} d u$. Here $J_{u_{1}+}^{0} \varsigma(x)=J_{\imath_{2}-}^{0} \varsigma(x)=\varsigma(x)$.

Note that $\alpha=1$, the fractional integral reduces to the classical integral.

In [33], Raina R. K. introduced a class of functions defined formally by

$$
\mathcal{F}_{\rho, \lambda}^{\sigma}(x)=\mathcal{F}_{\rho, \lambda}^{\sigma(0), \sigma(1), \ldots}(x)=\sum_{k=0}^{+\infty} \frac{\sigma(k)}{\Gamma(\rho k+\lambda)} x^{k},
$$

where $\rho, \lambda>0,|x|<R$ and $\sigma=(\sigma(0), \ldots, \sigma(k), \ldots)$ is a bounded sequence of positive real numbers. Note that, if we take in (1.6) $\rho=1, \lambda=0$ and $\sigma(k)=\frac{\left((\alpha)_{k}(\beta)_{k}\right)}{(\gamma)_{k}}$ for $k=0,1,2, \ldots$, where $\alpha, \beta$ and $\gamma$ are parameters which can take arbitrary real or complex values (provided that $\gamma \neq 0,-1,-2, \ldots$ ), and the symbol $(a)_{k}$ denote the quantity

$$
(a)_{k}=\frac{\Gamma(a+k)}{\Gamma(a)}=a(a+1) \ldots(a+k-1), \quad k=0,1,2, \ldots,
$$

and restrict its domain to $|x| \leq 1$ (with $x \in \mathbf{C}$ ), then we have the classical hypergeometric function, that is

$$
\mathcal{F}_{\rho, \lambda}^{\sigma}(x)=F(\alpha, \beta ; \gamma ; x)=\sum_{k=0}^{+\infty} \frac{(\alpha)_{k}(\beta)_{k}}{(\gamma)_{k} k !} x^{k} .
$$

Also, if $\sigma=(1,1, \ldots)$ with $\rho=\alpha,(\operatorname{Re}(\alpha)>0), \lambda=1$ and restricting its domain to $z \in \mathbf{C}$ in (1.6) then we have the classical Mittag-Leffler function

$$
E_{\alpha}(z)=\sum_{k=0}^{+\infty} \frac{1}{\Gamma(\alpha k+1)} z^{k} .
$$

Also, Noor in [23], introduced a generalized convex function concept as follows:

Let $O$ be a non empty closed set in a normed space $H$. We denote by $\langle\cdot, \cdot\rangle$ and $\|\cdot\|$ the inner product and norm respectively. Let $\phi: O \rightarrow \mathbf{R}$ be a continuous function. 
Definition 1.12. Let $u_{1} \in O$. The set $O$ is said to be $\phi$-convex set, if there exists a function $\phi$ such that $u_{1}+t e^{i \phi}\left(u_{1}-u_{2}\right) \in O$, for all $u_{1}, u_{2} \in O$ and $t \in[0,1]$.

With this definition also is introduced the following.

Definition 1.13. A function $\varsigma$ defined on $O$ is said to be $\phi$-convex if and only if there exists a function $\phi$ such that

$$
\varsigma\left(u_{1}+t e^{i \phi}\left(u_{1}-u_{2}\right)\right) \leq t \varsigma\left(u_{1}\right)+(1-t) \varsigma\left(u_{2}\right),
$$

for all $u_{1}, u_{2} \in O$ and $t \in[0,1]$.

Furthermore, let us define a function $\phi:[0, \infty) \rightarrow[0, \infty)$ satisfying the following conditions:

$$
\begin{gathered}
\int_{0}^{1} \frac{\phi(t)}{t} d t<\infty \\
\frac{1}{\mathbf{A}} \leq \frac{\phi(s)}{\phi(r)} \leq \mathbf{A} \text { for } \frac{1}{2} \leq \frac{s}{r} \leq 2 \\
\frac{\phi(r)}{r^{2}} \leq \mathbf{B} \frac{\phi(s)}{s^{2}} \text { for } s \leq r \\
\left|\frac{\phi(r)}{r^{2}}-\frac{\phi(s)}{s^{2}}\right| \leq \mathbf{C}|r-s| \frac{\phi(r)}{r^{2}} \text { for } \frac{1}{2} \leq \frac{s}{r} \leq 2
\end{gathered}
$$

where $\mathbf{A}, \mathbf{B}, \mathbf{C}>0$ are independent of $r, s>0$. If $\phi(r) r^{\alpha}$ is increasing for some $\alpha \geq 0$ and $\frac{\phi(r)}{r^{\beta}}$ is decreasing for some $\beta \geq 0$, then $\phi$ satisfies (1.7)(1.10), see reference [37]. Therefore, Sarikaya and Ertuğral [36] defined the following left-sided and right-sided generalized fractional integral operators, respectively, as follows:

$$
\begin{aligned}
& {\imath_{1}^{+}}_{\phi} I_{\phi}(x)=\int_{\imath_{1}}^{x} \frac{\phi(x-t)}{x-t} \varsigma(t) d t, \quad x>\imath_{1}, \\
& \imath_{2}^{-} I_{\phi} \varsigma(x)=\int_{x}^{\imath_{2}} \frac{\phi(t-x)}{t-x} \varsigma(t) d t, \quad x<\imath_{2} .
\end{aligned}
$$

This fractional integral operators are a new generalization of fractional integrals such as the Riemann-Liouville fractional integral, the $k$-RiemannLiouville fractional integral, Katugampola fractional integrals, the conformable fractional integral, Hadamard fractional integrals, etc. To read 
more about fractional analysis, see references [13, 14, 22, 35].

An important class of convex functions, which is called exponential convex functions, was introduced and studied by Antczak [2], Dragomir et al [9] and Noor et al [28]. Alirezai and Mathar [1] have investigated their basic properties along with their potential applications in statistics and information theory. Awan et al. [5] and Pecarić and Jaksetić [31] defined another kind of exponential convex functions and have shown that the class of exponential convex functions unifies various unrelated concepts.

Definition 1.14. $[2,9,27]$ A function $\varsigma: O \subseteq \mathbf{R} \rightarrow \mathbf{R}$ is said to be exponentially convex function, if

$$
e^{\varsigma\left((1-t) \imath_{1}+t \imath_{2}\right)} \leq(1-t) e^{\varsigma\left(\imath_{1}\right)}+t e^{\varsigma\left(\imath_{2}\right)}
$$

holds for all $\imath_{1}, \imath_{2} \in O, t \in[0,1]$, where $\varsigma$ is positive.

For the applications of exponentially convex functions and strongly exponentially convex functions in different field of statistics, information theory and mathematical sciences, see $[1,2,5],[24]-[28]$ and the references therein.

Definition 1.15. [34] A function $\varsigma: O \subseteq \mathbf{R} \rightarrow \mathbf{R}$ is said to be exponentially $m$-convex function, where $m \in(0,1]$, if

$$
e^{\varsigma\left((1-t) \imath_{1}+m t \imath_{2}\right)} \leq(1-t) e^{\varsigma\left(\imath_{1}\right)}+m t e^{\varsigma\left(\imath_{2}\right)}
$$

holds for all $\imath_{1}, \imath_{2} \in O, t \in[0,1]$, where $\varsigma$ is positive.

Motivated by the above literatures, the main objective of this article is to establish in Section 2 fractional integral inequalities using a new class of functions called strongly exponentially generalized $\left(m, \nu_{1}, \nu_{2}, g_{1}, g_{2}\right)$ with modulus $c$. Also, using a new identity pertaining differentiable functions defined on $m$-invex set as auxiliary result, some new Hermite-Hadamard inequalities for strongly exponentially generalized $\left(m, \nu_{1}, \nu_{2}, g_{1}, g_{2}\right)$ functions with modulus $c$ via Riemann-Liouville fractional integral will obtain. Also, some new estimates with respect to trapezium-type integral inequalities for strongly exponentially generalized $\left(m, \nu_{1}, \nu_{2}, g_{1}, g_{2}\right)$ functions with modulus $c$ via general fractional integrals will given. Various special cases will be discussed. In Section 3, some new error estimates for trapezoidal quadrature formula will be given. At the end, a briefly conclusion is given. 


\section{Main results}

Now, we are in a position to introduce a new class of functions so-called strongly exponentially generalized $\left(m, \nu_{1}, \nu_{2}, g_{1}, g_{2}\right)$ with modulus $c$ as follows:

Definition 2.1. Let $O=\left[m \imath_{1}, m \imath_{1}+\mathcal{F}_{\rho, \lambda}^{\sigma}\left(\imath_{2}-m \imath_{1}\right)\right] \subseteq \mathbf{R}$ be $m$-invex set for some fixed $m \in(0,1]$, where $\mathcal{F}_{\rho, \lambda}^{\sigma, \lambda}(\cdot)$ is defined from (1.6) and $g_{1}, g_{2}:[0,1] \rightarrow[0,+\infty)$. A function $\varsigma: O \rightarrow(0,+\infty)$ is called strongly exponentially generalized $\left(m, \nu_{1}, \nu_{2}, g_{1}, g_{2}\right)$ with modulus $c>0$, if

$e^{\varsigma\left(m \imath_{1}+t \mathcal{F}_{\rho, \lambda}^{\sigma}\left(\imath_{2}-m \imath_{1}\right)\right)} \leq m g_{1}(t) e^{\nu_{1} \varsigma\left(\imath_{1}\right)}+g_{2}(t) e^{\nu_{2} \varsigma\left(\imath_{2}\right)}-c m\left(\imath_{2}-\imath_{1}\right)^{2} g_{1}(t) g_{2}(t)$

holds for all $\imath_{1}, \imath_{2} \in O, t \in[0,1]$ and $\nu_{1}, \nu_{2} \in \mathbf{R}$.

Remark 2.2. In definition 2.1, taking $c \rightarrow 0^{+}$and choose $\nu_{1}=\nu_{2}=$ $1, g_{1}(t)=1-t, g_{2}(t)=t$, where $\mathcal{F}_{\rho, \lambda}^{\sigma}\left(\imath_{2}-m \imath_{1}\right)=\imath_{2}-m \imath_{1}$, this definition reduce to the definition 1.15 .

Remark 2.3. Under the conditions of remark 2.2, taking $m=1$, we get definition 1.14.

Remark 2.4. Let us discuss some special cases in definition 2.1 as follows:

(I) Taking $g_{1}(t)=g(1-t), g_{2}(t)=g(t)$, then we get strongly exponentially generalized $\left(m, \nu_{1}, \nu_{2}, g\right)$ functions with modulus $c$.

(II) Taking $g_{1}(t)=g_{2}(t)=t(1-t)$, then we get strongly exponentially generalized $\left(m, \nu_{1}, \nu_{2}, t g s\right)$ functions with modulus $c$.

(III) Taking $g_{1}(t)=\frac{\sqrt{1-t}}{2 \sqrt{t}}, g_{2}(t)=\frac{\sqrt{t}}{2 \sqrt{1-t}}$, then we get strongly exponentially generalized $\left(m, \nu_{1}, \nu_{2}\right)-M T$ functions with modulus $c$.

In this section, we obtain Hermite-Hadamard type inequalities for strongly exponentially generalized $\left(m, \nu_{1}, \nu_{2}, g_{1}, g_{2}\right)$ function with modulus $c$ via Riemann-Liouville fractional integral. 
Theorem 2.5. Let $O=\left[m \imath_{1}, m \imath_{1}+\mathcal{F}_{\rho, \lambda}^{\sigma}\left(\imath_{2}-m \imath_{1}\right)\right] \subseteq \mathbf{R}$ be $m$-invex set for some fixed $m \in(0,1]$, where $\imath_{1}<\imath_{2}$ and $\mathcal{F}_{\rho, \lambda}^{\sigma}\left(\imath_{2}-m \imath_{1}\right)>0$. Suppose $g_{1}, g_{2}:[0,1] \rightarrow[0,+\infty)$ be continuous functions. Let $\varsigma, \vartheta: O \rightarrow(0,+\infty)$ be strongly exponentially generalized $\left(m, \nu_{1}, \nu_{2}, g_{1}, g_{2}\right)$ functions with modulus $c>0$. If $\varsigma, \vartheta \in L(O)$, then for $\nu_{1}, \nu_{2} \in \mathbf{R}$ and $\alpha>0$, the following inequality holds:

$$
\begin{gathered}
\frac{\Gamma(\alpha)}{\left(F_{\rho, \lambda}^{\sigma}\left(i_{2}-m i_{2}\right)\right)^{\alpha}}\left\{J_{\left(m i_{1}+F_{\rho, \lambda}^{\sigma}\left(i_{2}-m i_{1}\right)\right)}^{\alpha}-e^{s\left(m i_{1}\right)}+J_{\left(m i_{1}+F_{\rho, \lambda}^{\sigma}\left(i_{2}-m i_{2}\right)\right)}^{\alpha}-e^{\vartheta\left(m i_{1}\right)}\right\} \\
(2.2) \leq m\left(e^{\nu_{1} \varsigma\left(\imath_{1}\right)}+e^{\nu_{1} \vartheta\left(\imath_{1}\right)}\right) H_{g_{1}}(\alpha)+\left(e^{\nu_{2} \varsigma\left(\imath_{2}\right)}+e^{\nu_{2} \vartheta\left(\imath_{2}\right)}\right) H_{g_{2}}(\alpha) \\
-2 c m\left(\imath_{2}-\imath_{1}\right)^{2} \Psi_{g_{1}, g_{2}}(\alpha),
\end{gathered}
$$

where

$$
\Psi_{g_{1}, g_{2}}(\alpha):=\int_{0}^{1} t^{\alpha-1} g_{1}(t) g_{2}(t) d t, \quad H_{g_{i}}(\alpha):=\int_{0}^{1} t^{\alpha-1} g_{i}(t) d t, \quad \forall i=1,2 .
$$

Proof. From strongly exponentially generalized $\left(m, \nu_{1}, \nu_{2}, g_{1}, g_{2}\right)$ with modulus $c$ of $\varsigma$ and $\vartheta$ for all $t \in[0,1]$, we have

$e^{\varsigma\left(m \imath_{1}+t \mathcal{F}_{\rho, \lambda}^{\sigma}\left(\imath_{2}-m \imath_{1}\right)\right)} \leq m g_{1}(t) e^{\nu_{1} \varsigma\left(\imath_{1}\right)}+g_{2}(t) e^{\nu_{2} \varsigma\left(\imath_{2}\right)}-c m\left(\imath_{2}-\imath_{1}\right)^{2} g_{1}(t) g_{2}(t)$

and

$e^{\vartheta\left(m \imath_{1}+t \mathcal{F}_{\rho, \lambda}^{\sigma}\left(\imath_{2}-m \imath_{1}\right)\right)} \leq m g_{1}(t) e^{\nu_{1} \vartheta\left(\imath_{1}\right)}+g_{2}(t) e^{\nu_{2} \vartheta\left(\imath_{2}\right)}-c m\left(\imath_{2}-\imath_{1}\right)^{2} g_{1}(t) g_{2}(t)$.

Adding both sides of the above inequalities, we get

$$
\begin{gathered}
e^{\varsigma\left(m a+t \mathcal{F}_{\rho, \lambda}^{\sigma}\left(\imath_{2}-m \imath_{1}\right)\right)}+e^{\vartheta\left(m \imath_{1}+t \mathcal{F}_{\rho, \lambda}^{\sigma}\left(\imath_{2}-m \imath_{1}\right)\right)} \leq m\left(e^{\nu_{1} \varsigma\left(\imath_{1}\right)}+e^{\nu_{1} \vartheta\left(\imath_{1}\right)}\right) g_{1}(t) \\
+\left(e^{\nu_{2} \varsigma\left(\imath_{2}\right)}+e^{\nu_{2} \vartheta\left(\imath_{2}\right)}\right) g_{2}(t)-2 c m\left(\imath_{2}-\imath_{1}\right)^{2} g_{1}(t) g_{2}(t) .
\end{gathered}
$$


Multiplying both sides of inequality (2.4) with $t^{\alpha-1}$ and integrating over $[0,1]$, we obtain

$$
\begin{gathered}
\int_{0}^{1} t^{\alpha-1}\left[e^{\varsigma\left(m \imath_{1}+t \mathcal{F}_{\rho, \lambda}^{\sigma}\left(\imath_{2}-m \imath_{1}\right)\right)}+e^{\vartheta\left(m \imath_{1}+t \mathcal{F}_{\rho, \lambda}^{\sigma}\left(\imath_{2}-m \imath_{1}\right)\right)}\right] d t \\
\leq m\left(e^{\nu_{1} \varsigma\left(\imath_{1}\right)}+e^{\nu_{1} \vartheta\left(\imath_{1}\right)}\right) \int_{0}^{1} t^{\alpha-1} g_{1}(t) d t+\left(e^{\nu_{2} \varsigma\left(\imath_{2}\right)}+e^{\nu_{2} \vartheta\left(\imath_{2}\right)}\right) \int_{0}^{1} t^{\alpha-1} g_{2}(t) d t \\
-2 c m\left(\imath_{2}-\imath_{1}\right)^{2} \int_{0}^{1} t^{\alpha-1} g_{1}(t) g_{2}(t) d t .
\end{gathered}
$$

Using definition 1.11, we get the required result.

Corollary 2.6. In Theorem 2.5, taking $c \rightarrow 0^{+}$, we get

$\frac{\Gamma(\alpha)}{\left(\mathcal{F}_{\rho, \lambda}^{\sigma}\left(\imath_{2}-m \imath_{1}\right)\right)^{\alpha}}\left\{J_{\left(m \imath_{1}+\mathcal{F}_{\rho, \lambda}^{\sigma}\left(\imath_{2}-m \imath_{1}\right)\right)^{-}}^{\alpha} e^{\varsigma\left(m \imath_{1}\right)}+J_{\left(m \imath_{1}+\mathcal{F}_{\rho, \lambda}^{\sigma}\left(\imath_{2}-m \imath_{1}\right)\right)^{-}}^{\alpha} e^{\vartheta\left(m \imath_{1}\right)}\right\}$

$$
\leq m\left(e^{\nu_{1} \varsigma\left(\imath_{1}\right)}+e^{\nu_{1} \vartheta\left(\imath_{1}\right)}\right) H_{g_{1}}(\alpha)+\left(e^{\nu_{2} \varsigma\left(\imath_{2}\right)}+e^{\nu_{2} \vartheta\left(\imath_{2}\right)}\right) H_{g_{2}}(\alpha) .
$$

Corollary 2.7. In Theorem 2.5, if we choose $m=1$ and $\mathcal{F}_{\rho, \lambda}^{\sigma}\left(\imath_{2}-m \imath_{1}\right)=$ $\imath_{2}-m \imath_{1}$, we have

$$
\begin{gathered}
\frac{\Gamma(\alpha)}{\left(\imath_{2}-\imath_{1}\right)^{\alpha}}\left\{J_{\imath_{2}^{-}}^{\alpha} e^{\varsigma\left(\imath_{1}\right)}+J_{\imath_{2}^{-}}^{\alpha} e^{\vartheta\left(\imath_{1}\right)}\right\} \\
\leq\left(e^{\nu_{1} \varsigma\left(\imath_{1}\right)}+e^{\nu_{1} \vartheta\left(\imath_{1}\right)}\right) H_{g_{1}}(\alpha)+\left(e^{\nu_{2} \varsigma\left(\imath_{2}\right)}+e^{\nu_{2} \vartheta\left(\imath_{2}\right)}\right) H_{g_{2}}(\alpha)-2 c\left(\imath_{2}-\imath_{1}\right)^{2} \Psi_{g_{1}, g_{2}}(\alpha) .
\end{gathered}
$$

Corollary 2.8. In Theorem 2.5, if we choose $\alpha=1$, we obtain

$$
\begin{gathered}
\frac{1}{\mathcal{F}_{\rho, \lambda}^{\sigma}\left(\imath_{2}-m \imath_{1}\right)} \int_{m \imath_{1}}^{m \imath_{1}+\mathcal{F}_{\rho, \lambda}^{\sigma}\left(\imath_{2}-m \imath_{1}\right)}\left[e^{\varsigma(t)}+e^{\vartheta(t)}\right] d t \\
\leq m\left(e^{\nu_{1} \varsigma\left(\imath_{1}\right)}+e^{\nu_{1} \vartheta\left(\imath_{1}\right)}\right) H_{g_{1}}+\left(e^{\nu_{2} \varsigma\left(\imath_{2}\right)}+e^{\nu_{2} \vartheta\left(\imath_{2}\right)}\right) H_{g_{2}}-2 c m\left(\imath_{2}-\imath_{1}\right)^{2} F_{g_{1}, g_{2}},
\end{gathered}
$$

where

$$
F_{g_{1}, g_{2}}:=\int_{0}^{1} g_{1}(t) g_{2}(t) d t, \quad H_{g_{i}}:=\int_{0}^{1} g_{i}(t) d t, \quad \forall i=1,2 .
$$


Theorem 2.9. Let $O=\left[m \imath_{1}, m \imath_{1}+\mathcal{F}_{\rho, \lambda}^{\sigma}\left(\imath_{2}-m \imath_{1}\right)\right] \subseteq \mathbf{R}$ be $m$-invex set for some fixed $m \in(0,1]$, where $\imath_{1}<\imath_{2}$ and $\mathcal{F}_{\rho, \lambda}^{\sigma}\left(\imath_{2}-m \imath_{1}\right)>0$. Suppose $g_{1}, g_{2}:[0,1] \rightarrow[0,+\infty)$ be continuous functions. Let $\varsigma, \vartheta: O \rightarrow(0,+\infty)$ be strongly exponentially generalized ( $\left.m, \nu_{1}, \nu_{2}, g_{1}, g_{2}\right)$ functions with modulus $c>0$. If $\varsigma, \vartheta \in L(O)$, then for $\nu_{1}, \nu_{2} \in \mathbf{R}$ and $\alpha>0$, the following inequality holds:

$\frac{\Gamma(\alpha)}{\left(\mathcal{F}_{\rho, \lambda}^{\sigma}\left(\imath_{2}-m \imath_{1}\right)\right)^{\alpha}}\left\{J_{\left(m \imath_{1}\right)^{+}}^{\alpha} e^{\varsigma\left(m \imath_{1}+\mathcal{F}_{\rho, \lambda}^{\sigma}\left(\imath_{2}-m \imath_{1}\right)\right)}+J_{\left(m \imath_{1}+\mathcal{F}_{\rho, \lambda}^{\sigma}\left(\imath_{2}-m \imath_{1}\right)\right)^{-}}^{\alpha} e^{\vartheta\left(m \iota_{1}\right)}\right\}$

$(2.9) \leq m\left(e^{\nu_{1} \varsigma\left(\imath_{1}\right)} C_{g_{1}}(\alpha)+e^{\nu_{1} \vartheta\left(\imath_{1}\right)} H_{g_{1}}(\alpha)\right)+e^{\nu_{2} \varsigma\left(\imath_{2}\right)} C_{g_{2}}(\alpha)+e^{\nu_{2} \vartheta\left(\imath_{2}\right)} H_{g_{2}}(\alpha)$

$$
-c m\left(\imath_{2}-\imath_{1}\right)^{2} D_{g_{1}, g_{2}}(\alpha)
$$

where

$$
\begin{aligned}
D_{g_{1}, g_{2}}(\alpha) & :=\int_{0}^{1}\left[t^{\alpha}+(1-t)^{\alpha-1}\right] g_{1}(t) g_{2}(t) d t, \\
C_{g_{i}}(\alpha) & :=\int_{0}^{1}(1-t)^{\alpha-1} g_{i}(t) d t, \quad \forall i=1,2
\end{aligned}
$$

and $H_{g_{1}}(\alpha), H_{g_{2}}(\alpha)$ are defined as in Theorem 2.5.

Proof. From strongly exponentially generalized $\left(m, \nu_{1}, \nu_{2}, g_{1}, g_{2}\right)$ with modulus $c$ of $\varsigma$ and $\vartheta$ for all $t \in[0,1]$, we have

$e^{\varsigma\left(m \imath_{1}+t \mathcal{F}_{\rho, \lambda}^{\sigma}\left(\imath_{2}-m \imath_{1}\right)\right)} \leq m g_{1}(t) e^{\nu_{1} \varsigma\left(\imath_{1}\right)}+g_{2}(t) e^{\nu_{2} \varsigma\left(\imath_{2}\right)}-c m\left(\imath_{2}-\imath_{1}\right)^{2} g_{1}(t) g_{2}(t)$

and

$e^{\vartheta\left(m \imath_{1}+t \mathcal{F}_{\rho, \lambda}^{\sigma}\left(\imath_{2}-m \imath_{1}\right)\right)} \leq m g_{1}(t) e^{\nu_{1} \vartheta\left(\imath_{1}\right)}+g_{2}(t) e^{\nu_{2} \vartheta\left(\imath_{2}\right)}-c m\left(\imath_{2}-\imath_{1}\right)^{2} g_{1}(t) g_{2}(t)$.

Multiplying first above inequality with $(1-t)^{\alpha-1}$, the second with $t^{\alpha-1}$ and adding both sides, we get

$$
(1-t)^{\alpha-1} e^{\varsigma\left(m \imath_{1}+t \mathcal{F}_{\rho, \lambda}^{\sigma}\left(\imath_{2}-m \imath_{1}\right)\right)}+t^{\alpha-1} e^{\vartheta\left(m \imath_{1}+t \mathcal{F}_{\rho, \lambda}^{\sigma}\left(\imath_{2}-m \imath_{1}\right)\right)}
$$

$$
+t^{\alpha-1}\left[m g_{1}(t) e^{\nu_{1} \vartheta\left(\imath_{1}\right)}+g_{2}(t) e^{\nu_{2} \vartheta\left(\imath_{2}\right)}\right]-c m\left(\imath_{2}-\imath_{1}\right)^{2} \int_{0}^{1}\left[t^{\alpha}+(1-t)^{\alpha-1}\right] g_{1}(t) g_{2}(t) d t \text {. }
$$

Integrating over $[0,1]$ both sides of inequality (2.12) and using definition 1.11 , we get the required result. 
Corollary 2.10. In Theorem 2.9, taking $c \rightarrow 0^{+}$, we get

$\frac{\Gamma(\alpha)}{\left(\mathcal{F}_{\rho, \lambda}^{\sigma}\left(\imath_{2}-m \imath_{1}\right)\right)^{\alpha}}\left\{J_{\left(m \imath_{1}\right)^{+}}^{\alpha} e^{\varsigma\left(m \imath_{1}+\mathcal{F}_{\rho, \lambda}^{\sigma}\left(\imath_{2}-m \imath_{1}\right)\right)}+J_{\left(m \imath_{1}+\mathcal{F}_{\rho, \lambda}^{\sigma}\left(\imath_{2}-m \imath_{1}\right)\right)^{-}}^{\alpha} e^{\vartheta\left(m \imath_{1}\right)}\right\}$

$(2.13) \leq m\left(e^{\nu_{1} \varsigma\left(\imath_{1}\right)} C_{g_{1}}(\alpha)+e^{\nu_{1} \vartheta\left(\imath_{1}\right)} H_{g_{1}}(\alpha)\right)+e^{\nu_{2} \varsigma\left(\imath_{2}\right)} C_{g_{2}}(\alpha)+e^{\nu_{2} \vartheta\left(\imath_{2}\right)} H_{g_{2}}(\alpha)$.

Corollary 2.11. In Theorem 2.9, if we choose $m=1$ and $\mathcal{F}_{\rho, \lambda}^{\sigma}\left(\imath_{2}-m \imath_{1}\right)=$ $\imath_{2}-m \imath_{1}$, we have

$$
\frac{\Gamma(\alpha)}{\left(\imath_{2}-\imath_{1}\right)^{\alpha}}\left\{J_{\imath_{1}^{+}}^{\alpha} e^{\varsigma\left(\imath_{2}\right)}+J_{\imath_{2}^{-}}^{\alpha} e^{\vartheta\left(\imath_{1}\right)}\right\}
$$

$\left(2.14 £ e^{\nu_{1} \varsigma\left(\imath_{1}\right)} C_{g_{1}}(\alpha)+e^{\nu_{1} \vartheta\left(\imath_{1}\right)} H_{g_{1}}(\alpha)+e^{\nu_{2} \varsigma\left(\imath_{2}\right)} C_{g_{2}}(\alpha)+e^{\nu_{2} \vartheta\left(\imath_{2}\right)} H_{g_{2}}(\alpha)\right.$

$$
-c\left(\imath_{2}-\imath_{1}\right)^{2} D_{g_{1}, g_{2}}(\alpha) \text {. }
$$

Corollary 2.12. In Theorem 2.9, if we choose $\alpha=1$, we obtain Corollary 2.8 .

Remark 2.13. Under the conditions of Theorems 2.5 and 2.9, using remark 2.4, we can get several new integral inequalities. The details are left to the interested reader.

For establishing some new results regarding generalizations of HermiteHadamard type integral inequalities associated with strongly exponentially generalized $\left(m, \nu_{1}, \nu_{2}, g_{1}, g_{2}\right)$ with modulus $c$ via general fractional integrals, we need the following lemma.

Lemma 2.14. Let $O=\left[m \imath_{1}, m \imath_{1}+\mathcal{F}_{\rho, \lambda}^{\sigma}\left(\imath_{2}-m \imath_{1}\right)\right] \subseteq \mathbf{R}$ be $m$-invex set for some fixed $m \in(0,1]$, where $\imath_{1}<\imath_{2}$ and $\mathcal{F}_{\rho, \lambda}^{\sigma}\left(\imath_{2}-m \imath_{1}\right)>0$. If $\varsigma: O \rightarrow \mathbf{R}$ is a differentiable function on $O^{\circ}$ such that $\varsigma^{\prime} \in L(O)$, then the following identity for generalized fractional integrals holds:

$$
\begin{gathered}
\frac{e^{\varsigma\left(m \imath_{1}\right)}+e^{\varsigma\left(m \imath_{1}+\mathcal{F}_{\rho, \lambda}^{\sigma}\left(\imath_{2}-m \imath_{1}\right)\right)}}{2}-\frac{1}{2 \Pi_{m}(1) \mathcal{F}_{\rho, \lambda}^{\sigma}\left(\imath_{2}-m \imath_{1}\right)} \\
\times\left\{\left(m \imath_{1}\right)^{+} I_{\phi} e^{\varsigma\left(m \imath_{1}+\mathcal{F}_{\rho, \lambda}^{\sigma}\left(\imath_{2}-m \imath_{1}\right)\right)}+{ }_{\left(m \imath_{1}+\mathcal{F}_{\rho, \lambda}^{\sigma}\left(\imath_{2}-m \imath_{1}\right)\right)} I_{\phi} e^{\varsigma\left(m \imath_{1}\right)}\right\}
\end{gathered}
$$




$$
=\frac{\mathcal{F}_{\rho, \lambda}^{\sigma}\left(\imath_{2}-m \imath_{1}\right)}{2 \Pi_{m}(1)}
$$

$\times \int_{0}^{1}\left[\Pi_{m}(t)-\Pi_{m}(1-t)\right] e^{\varsigma\left(m \imath_{1}+t \mathcal{F}_{\rho, \lambda}^{\sigma}\left(\imath_{2}-m \imath_{1}\right)\right)} \varsigma^{\prime}\left(m \imath_{1}+t \mathcal{F}_{\rho, \lambda}^{\sigma}\left(\imath_{2}-m \imath_{1}\right)\right) d t$,

where

$$
\Pi_{m}(t):=\int_{0}^{t} \frac{\phi\left(\mathcal{F}_{\rho, \lambda}^{\sigma}\left(\imath_{2}-m \imath_{1}\right) u\right)}{u} d u<\infty
$$

We denote

$$
\Xi_{\varsigma, \Pi_{m}}\left(\imath_{1}, \imath_{2}\right):=\frac{\mathcal{F}_{\rho, \lambda}^{\sigma}\left(\imath_{2}-m \imath_{1}\right)}{2 \Pi_{m}(1)}
$$

$$
\times \int_{0}^{1}\left[\Pi_{m}(t)-\Pi_{m}(1-t)\right] e^{\varsigma\left(m \imath_{1}+t \mathcal{F}_{\rho, \lambda}^{\sigma}\left(\imath_{2}-m \imath_{1}\right)\right)} \varsigma^{\prime}\left(m \imath_{1}+t \mathcal{F}_{\rho, \lambda}^{\sigma}\left(\imath_{2}-m \imath_{1}\right)\right) d t
$$

Proof. From (2.17), we have

$$
\begin{gathered}
\Xi_{\varsigma, \Pi_{m}}\left(\imath_{1}, \imath_{2}\right)=\frac{\mathcal{F}_{\rho, \lambda}^{\sigma}\left(\imath_{2}-m \imath_{1}\right)}{2 \Pi_{m}(1)} \\
\times\left[\int_{0}^{1} \Pi_{m}(t) e^{\varsigma\left(m \imath_{1}+t \mathcal{F}_{\rho, \lambda}^{\sigma}\left(\imath_{2}-m \imath_{1}\right)\right)} \varsigma^{\prime}\left(m \imath_{1}+t \mathcal{F}_{\rho, \lambda}^{\sigma}\left(\imath_{2}-m \imath_{1}\right)\right) d t\right. \\
\left.-\int_{0}^{1} \Pi_{m}(1-t) e^{\varsigma\left(m \imath_{1}+t \mathcal{F}_{\rho, \lambda}^{\sigma}\left(\imath_{2}-m \imath_{1}\right)\right)} \varsigma^{\prime}\left(m \imath_{1}+t \mathcal{F}_{\rho, \lambda}^{\sigma}\left(\imath_{2}-m \imath_{1}\right)\right) d t\right] \\
=\frac{\mathcal{F}_{\rho, \lambda}^{\sigma}\left(\imath_{2}-m \imath_{1}\right)}{2} \times\left[\Xi_{\varsigma, \Pi_{m}}^{(1)}\left(\imath_{1}, \imath_{2}\right)-\Xi_{\varsigma, \Pi_{m}}^{(2)}\left(\imath_{1}, \imath_{2}\right)\right],
\end{gathered}
$$

where

(2.19) $\Xi_{\varsigma, \Pi_{m}}^{(1)}\left(\imath_{1}, \imath_{2}\right):=\int_{0}^{1} \Pi_{m}(t) e^{\varsigma\left(m \iota_{1}+t \mathcal{F}_{\rho, \lambda}^{\sigma}\left(\imath_{2}-m \imath_{1}\right)\right)} \varsigma^{\prime}\left(m \imath_{1}+t \mathcal{F}_{\rho, \lambda}^{\sigma}\left(\imath_{2}-m \imath_{1}\right)\right) d t$ and 
$\Xi_{\varsigma, \Pi_{m}}^{(2)}\left(\imath_{1}, \imath_{2}\right):=\int_{0}^{1} \Pi_{m}(1-t) e^{\varsigma\left(m \imath_{1}+t \mathcal{F}_{\rho, \lambda}^{\sigma}\left(\imath_{2}-m \imath_{1}\right)\right)} \varsigma^{\prime}\left(m \imath_{1}+t \mathcal{F}_{\rho, \lambda}^{\sigma}\left(\imath_{2}-m \imath_{1}\right)\right) d t$.

Now, integrating by parts (2.19), changing the variable $u=m \imath_{1}+$ $t \mathcal{F}_{\rho, \lambda}^{\sigma}\left(\imath_{2}-m \imath_{1}\right)$ and using definition 1.11 , we get

$$
\begin{gathered}
\Xi_{\varsigma, \Pi_{m}}^{(1)}\left(\imath_{1}, \imath_{2}\right):=\left.\frac{\Pi_{m}(t) e^{\varsigma\left(m \imath_{1}+t \mathcal{F}_{\rho, \lambda}^{\sigma}\left(\imath_{2}-m \imath_{1}\right)\right)}}{\mathcal{F}_{\rho, \lambda}^{\sigma}\left(\imath_{2}-m \imath_{1}\right)}\right|_{0} ^{1} \\
-\frac{1}{\mathcal{F}_{\rho, \lambda}^{\sigma}\left(\imath_{2}-m \imath_{1}\right)} \int_{0}^{1} \frac{\phi\left(\mathcal{F}_{\rho, \lambda}^{\sigma}\left(\imath_{2}-m \imath_{1}\right) t\right)}{t} e^{\varsigma\left(m \imath_{1}+t \mathcal{F}_{\rho, \lambda}^{\sigma}\left(\imath_{2}-m \imath_{1}\right)\right)} d t \\
=\frac{\Pi_{m}(1) e^{\varsigma\left(m \imath_{1}+\mathcal{F}_{\rho, \lambda}^{\sigma}\left(\imath_{2}-m \imath_{1}\right)\right)}}{\mathcal{F}_{\rho, \lambda}^{\sigma}\left(\imath_{2}-m \imath_{1}\right)}-\frac{1}{\mathcal{F}_{\rho, \lambda}^{\sigma, 2}\left(\imath_{2}-m \imath_{1}\right)}\left(m \iota_{1}+\mathcal{F}_{\rho, \lambda}^{\sigma}\left(\imath_{2}-m \imath_{1}\right)\right)^{-} I_{\phi} e^{\varsigma\left(m \iota_{1}\right)} .
\end{gathered}
$$

Similarly, using (2.20), we obtain

$\Xi_{\varsigma, \Pi_{m}}^{(2)}\left(\imath_{1}, \imath_{2}\right)=-\frac{\Pi_{m}(1) e^{\varsigma\left(m \imath_{1}\right)}}{\mathcal{F}_{\rho, \lambda}^{\sigma}\left(\imath_{2}-m \imath_{1}\right)}+\frac{1}{\mathcal{F}_{\rho, \lambda}^{\sigma, 2}\left(\imath_{2}-m \imath_{1}\right)}\left(m \imath_{1}\right)^{+} I_{\phi} e^{\varsigma\left(m \imath_{1}+\mathcal{F}_{\rho, \lambda}^{\sigma}\left(\imath_{2}-m \imath_{1}\right)\right)}$.

Substituting (2.21) and (2.22) in (2.18), we get (2.15). The proof of Lemma 2.14 is completed.

Remark 2.15. In Lemma 2.14, if we choose $\phi(t)=\frac{t^{\alpha}}{\Gamma(\alpha)}$ for $\alpha>0$, we have

$$
\begin{gathered}
\frac{e^{\varsigma\left(m \imath_{1}\right)}+e^{\varsigma\left(m \imath_{1}+\mathcal{F}_{\rho, \lambda}^{\sigma}\left(\imath_{2}-m \imath_{1}\right)\right)}}{2} \\
-\frac{\Gamma(\alpha+1)}{2 \mathcal{F}_{\rho, \lambda}^{\sigma, \alpha}\left(\imath_{2}-m \imath_{1}\right)} \times\left\{J_{\left(m \imath_{1}\right)^{+}}^{\alpha} e^{\varsigma\left(m \imath_{1}+\mathcal{F}_{\rho, \lambda}^{\sigma}\left(\imath_{2}-m \imath_{1}\right)\right)}+J_{\left(m \imath_{1}+\mathcal{F}_{\rho, \lambda}^{\sigma}\left(\imath_{2}-m \imath_{1}\right)\right)^{-}}^{\alpha} e^{\varsigma\left(m \imath_{1}\right)}\right\} \\
=\frac{\mathcal{F}_{\rho, \lambda}^{\sigma}\left(\imath_{2}-m \imath_{1}\right)}{2} \int_{0}^{1}\left[t^{\alpha}-(1-t)^{\alpha}\right] e^{\varsigma\left(m \imath_{1}+t \mathcal{F}_{\rho, \lambda}^{\sigma}\left(\imath_{2}-m \imath_{1}\right)\right)} \varsigma^{\prime}\left(m \imath_{1}+t \mathcal{F}_{\rho, \lambda}^{\sigma}\left(\imath_{2}-m \imath_{1}\right)\right) d t .
\end{gathered}
$$

Using Lemma 2.14, we now state the following theorem. 
Theorem 2.16. Let $O=\left[m \imath_{1}, m \imath_{1}+\mathcal{F}_{\rho, \lambda}^{\sigma}\left(\imath_{2}-m \imath_{1}\right)\right] \subseteq \mathbf{R}$ be $m$-invex set for some fixed $m \in(0,1]$, where $\imath_{1}<\imath_{2}$ and $\mathcal{F}_{\rho, \lambda}^{\sigma}\left(\imath_{2}-m \imath_{1}\right)>0$. Suppose $g_{1}, g_{2}:[0,1] \rightarrow[0,+\infty)$ be continuous functions. Let $\varsigma: O \rightarrow(0,+\infty)$ be a differentiable strongly exponentially generalized $\left(m, \nu_{1}, \nu_{2}, g_{1}, g_{2}\right)$ function with modulus $c>0$ on $O^{\circ}$ such that $\varsigma^{\prime} \in L(O)$ and $\nu_{1}, \nu_{2} \in \mathbf{R}$. If $\left|\varsigma^{\prime}\right|^{q}$ is generalized $\left(m, g_{1}, g_{2}\right)$-preinvex function, then for $q>1$ and $\frac{1}{p}+\frac{1}{q}=1$, the following inequality holds:

$$
\begin{gathered}
(2.24) \quad\left|\Xi_{\varsigma, \Pi_{m}}\left(\imath_{1}, \imath_{2}\right)\right| \leq \frac{\mathcal{F}_{\rho, \lambda}^{\sigma}\left(\imath_{2}-m \imath_{1}\right)}{2 \Pi_{m}(1)} \sqrt[p]{B_{\Pi_{m}}(p)} \\
\times\left[m^{2} e^{q \nu_{1} \varsigma\left(\imath_{1}\right)}\left|\varsigma^{\prime}\left(\imath_{1}\right)\right|^{q} G_{g_{1}}+m \Delta_{\varsigma}\left(q ; \nu_{1}, \nu_{2}, \imath_{1}, \imath_{2}\right) F_{g_{1}, g_{2}}+e^{q \nu_{2} \varsigma\left(\imath_{2}\right)}\left|\varsigma^{\prime}\left(\imath_{2}\right)\right|^{q} G_{g_{2}}\right. \\
\left.-c m\left(\imath_{2}-\imath_{1}\right)^{2}\left(m\left|\varsigma^{\prime}\left(\imath_{1}\right)\right|^{q} M_{g_{1}, g_{2}}+\left|\varsigma^{\prime}\left(\imath_{2}\right)\right|^{q} N_{g_{1}, g_{2}}\right)\right]^{\frac{1}{q}}
\end{gathered}
$$

where

$$
\begin{gathered}
B_{\Pi_{m}}(p):=\int_{0}^{1}\left|\Pi_{m}(t)-\Pi_{m}(1-t)\right|^{p} d t, \quad G_{g_{i}}:=\int_{0}^{1}\left[g_{i}(t)\right]^{2} d t, \quad \forall i=1,2, \\
M_{g_{1}, g_{2}}:=\int_{0}^{1}\left[g_{1}(t)\right]^{2} g_{2}(t) d t, \quad N_{g_{1}, g_{2}}:=\int_{0}^{1} g_{1}(t)\left[g_{2}(t)\right]^{2} d t \\
\Delta_{\varsigma}\left(q ; \nu_{1}, \nu_{2}, \imath_{1}, \imath_{2}\right):=e^{q \nu_{1} \varsigma\left(\imath_{1}\right)}\left|\varsigma^{\prime}\left(\imath_{2}\right)\right|^{q}+e^{q \nu_{2} \varsigma\left(\imath_{2}\right)}\left|\varsigma^{\prime}\left(\imath_{1}\right)\right|^{q}
\end{gathered}
$$

and $F_{g_{1}, g_{2}}$ is defined from (2.8).

Proof. From Lemma 2.14, strongly exponentially generalized $\left(m, \nu_{1}, \nu_{2}, g_{1}, g_{2}\right)$ with modulus $c$ of $\varsigma$, generalized $\left(m, g_{1}, g_{2}\right)$-preinvexity of $\left|\varsigma^{\prime}\right|^{q}$, Hölder inequality and properties of the modulus, we have

$$
\begin{gathered}
\left|\Xi_{\varsigma, \Pi_{m}}\left(\imath_{1}, \imath_{2}\right)\right| \leq \frac{\mathcal{F}_{\rho, \lambda}^{\sigma}\left(\imath_{2}-m \imath_{1}\right)}{2 \Pi_{m}(1)} \\
\times \int_{0}^{1}\left|\Pi_{m}(t)-\Pi_{m}(1-t)\right|\left|e^{\varsigma\left(m \imath_{1}+t \mathcal{F}_{\rho, \lambda}^{\sigma}\left(\imath_{2}-m \imath_{1}\right)\right)} \varsigma^{\prime}\left(m \imath_{1}+t \mathcal{F}_{\rho, \lambda}^{\sigma}\left(\imath_{2}-m \imath_{1}\right)\right)\right| d t \\
\leq \frac{\mathcal{F}_{\rho, \lambda}^{\sigma}\left(\imath_{2}-m \imath_{1}\right)}{2 \Pi_{m}(1)} \sqrt[p]{B_{\Pi_{m}}(p)}\left(\int_{0}^{1} e^{q \varsigma\left(m \imath_{1}+t \mathcal{F}_{\rho, \lambda}^{\sigma}\left(\imath_{2}-m \imath_{1}\right)\right)}\left|\varsigma^{\prime}\left(m \imath_{1}+t \mathcal{F}_{\rho, \lambda}^{\sigma}\left(\imath_{2}-m \imath_{1}\right)\right)\right|^{q} d t\right)^{\frac{1}{q}}
\end{gathered}
$$




$$
\begin{gathered}
\leq \frac{\mathcal{F}_{\rho, \lambda}^{\sigma}\left(\imath_{2}-m \imath_{1}\right)}{2 \Pi_{m}(1)} \sqrt[p]{B_{\Pi_{m}}(p)} \\
\times\left\{\int_{0}^{1}\left[m g_{1}(t) e^{q \nu_{1} \varsigma\left(\imath_{1}\right)}+g_{2}(t) e^{q \nu_{2} \varsigma\left(\imath_{2}\right)}-c m\left(\imath_{2}-\imath_{1}\right)^{2} g_{1}(t) g_{2}(t)\right]\right. \\
\left.\times\left[m g_{1}(t)\left|\varsigma^{\prime}\left(\imath_{1}\right)\right|^{q}+g_{2}(t)\left|\varsigma^{\prime}\left(\imath_{2}\right)\right|^{q}\right] d t\right\}^{\frac{1}{q}} \\
=\frac{\mathcal{F}_{\rho, \lambda}^{\sigma}\left(\imath_{2}-m \imath_{1}\right)}{2 \Pi_{m}(1)} \sqrt[p]{B_{\Pi_{m}}(p)} \\
\times\left[m^{2} e^{q \nu_{1} \varsigma\left(\imath_{1}\right)\left|\varsigma^{\prime}\left(\imath_{1}\right)\right|^{q} G_{g_{1}}+m \Delta_{f}\left(q ; \nu_{1}, \nu_{2}, \imath_{1}, \imath_{2}\right) F_{g_{1}, g_{2}}+e^{q \nu_{2} \varsigma\left(\imath_{2}\right)}\left|\varsigma^{\prime}\left(\imath_{2}\right)\right|^{q} G_{g_{2}}}\right. \\
\left.-c m\left(\imath_{2}-\imath_{1}\right)^{2}\left(m\left|\varsigma^{\prime}\left(\imath_{1}\right)\right|^{q} M_{g_{1}, g_{2}}+\left|\varsigma^{\prime}\left(\imath_{2}\right)\right|^{q} N_{g_{1}, g_{2}}\right)\right]^{\frac{1}{q}} .
\end{gathered}
$$

The proof of Theorem 2.16 is completed.

We point out some special cases of Theorem 2.16.

Corollary 2.17. In Theorem 2.16, taking $c \rightarrow 0^{+}$, we get

$$
\left|\Xi_{f, \Pi_{m}}\left(\imath_{1}, \imath_{2}\right)\right| \leq \frac{\mathcal{F}_{\rho, \lambda}^{\sigma}\left(\imath_{2}-m \imath_{1}\right)}{2 \Pi_{m}(1)} \sqrt[p]{B_{\Pi_{m}}(p)}
$$

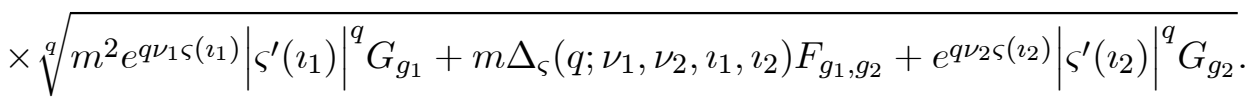

Corollary 2.18. In Theorem 2.16, if we choose $\phi(t)=t$, we have

$$
\begin{aligned}
& \left|\frac{e^{\varsigma\left(m \imath_{1}\right)}+e^{\varsigma\left(m \imath_{1}+\mathcal{F}_{\rho, \lambda}^{\sigma}\left(\imath_{2}-m \imath_{1}\right)\right)}}{2}-\frac{1}{\mathcal{F}_{\rho, \lambda}^{\sigma}\left(\imath_{2}-m \imath_{1}\right)} \int_{m \imath_{1}}^{m \imath_{1}+\mathcal{F}_{\rho, \lambda}^{\sigma}\left(\imath_{2}-m \imath_{1}\right)} e^{\varsigma(t)} d t\right| \\
& \leq \frac{\mathcal{F}_{\rho, \lambda}^{\sigma}\left(\imath_{2}-m \imath_{1}\right)}{2 \sqrt[p]{p+1}} \\
& \times\left[m^{2} e^{q \nu_{1} \varsigma\left(\imath_{1}\right)}\left|\varsigma^{\prime}\left(\imath_{1}\right)\right|^{q} G_{g_{1}}+m \Delta_{\varsigma}\left(q ; \nu_{1}, \nu_{2}, \imath_{1}, \imath_{2}\right) F_{g_{1}, g_{2}}+e^{q \nu_{2} \varsigma\left(\imath_{2}\right)}\left|\varsigma^{\prime}\left(\imath_{2}\right)\right|^{q} G_{g_{2}}\right. \\
& \left.-c m\left(\imath_{2}-\imath_{1}\right)^{2}\left(m\left|\varsigma^{\prime}\left(\imath_{1}\right)\right|^{q} M_{g_{1}, g_{2}}+\left|\varsigma^{\prime}\left(\imath_{2}\right)\right|^{q} N_{g_{1}, g_{2}}\right)\right]^{\frac{1}{q}} .
\end{aligned}
$$


Corollary 2.19. In Theorem 2.16, if we choose $\phi(t)=\frac{t^{\alpha}}{\Gamma(\alpha)}$ for $\alpha>0$ and $\mathcal{F}_{\rho, \lambda}^{\sigma}\left(\imath_{2}-m \imath_{1}\right)=\imath_{2}-m \imath_{1}$, we obtain

$$
\begin{gathered}
\left|\frac{e^{\varsigma\left(m \imath_{1}\right)}+e^{\varsigma\left(\imath_{2}\right)}}{2}-\frac{\Gamma(\alpha+1)}{2\left(\imath_{2}-m \imath_{1}\right)^{\alpha}} \times\left\{J_{\left(m \imath_{1}\right)^{+}}^{\alpha} e^{\varsigma\left(\imath_{2}\right)}+J_{\imath_{2}^{-}}^{\alpha} e^{\varsigma\left(m \imath_{1}\right)}\right\}\right| \\
\leq \frac{\left(\imath_{2}-m \imath_{1}\right)}{2} \sqrt[p]{B(p, \alpha)}
\end{gathered}
$$

$$
\begin{gathered}
\times\left[m^{2} e^{q \nu_{1} \varsigma\left(\imath_{1}\right)}\left|\varsigma^{\prime}\left(\imath_{1}\right)\right|^{q} G_{g_{1}}+m \Delta_{\varsigma}\left(q ; \nu_{1}, \nu_{2}, \imath_{1}, \imath_{2}\right) F_{g_{1}, g_{2}}+e^{q \nu_{2} \varsigma\left(\imath_{2}\right)}\left|\varsigma^{\prime}\left(\imath_{2}\right)\right|^{q} G_{g_{2}}\right. \\
\left.-c m\left(\imath_{2}-\imath_{1}\right)^{2}\left(m\left|\varsigma^{\prime}\left(\imath_{1}\right)\right|^{q} M_{g_{1}, g_{2}}+\left|\varsigma^{\prime}\left(\imath_{2}\right)\right|^{q} N_{g_{1}, g_{2}}\right)\right]^{\frac{1}{q}}
\end{gathered}
$$

where

$$
B(p, \alpha):=\int_{0}^{1}\left|t^{\alpha}-(1-t)^{\alpha}\right|^{p} d t .
$$

Theorem 2.20. Let $O=\left[m \imath_{1}, m \imath_{1}+\mathcal{F}_{\rho, \lambda}^{\sigma}\left(\imath_{2}-m \imath_{1}\right)\right] \subseteq \mathbf{R}$ be $m$-invex set for some fixed $m \in(0,1]$, where $\imath_{1}<\imath_{2}$ and $\mathcal{F}_{\rho, \lambda}^{\sigma}\left(\imath_{2}-m \imath_{1}\right)>0$. Suppose $g_{1}, g_{2}:[0,1] \rightarrow[0,+\infty)$ be continuous functions. Let $\varsigma: O \rightarrow(0,+\infty)$ be a differentiable strongly exponentially generalized $\left(m, \nu_{1}, \nu_{2}, g_{1}, g_{2}\right)$ function with modulus $c>0$ on $O^{\circ}$ such that $\varsigma^{\prime} \in L(O)$ and $\nu_{1}, \nu_{2} \in \mathbf{R}$. If $\left|\varsigma^{\prime}\right|^{q}$ is generalized ( $\left.m, g_{1}, g_{2}\right)$-preinvex function, then for $q \geq 1$, the following inequality holds:

$$
\left|\Xi_{\varsigma, \Pi_{m}}\left(\imath_{1}, \imath_{2}\right)\right| \leq \frac{\mathcal{F}_{\rho, \lambda}^{\sigma}\left(\imath_{2}-m \imath_{1}\right)}{2 \Pi_{m}(1)}\left[B_{\Pi_{m}}(1)\right]^{1-\frac{1}{q}}
$$

$$
\begin{gathered}
\times\left[m^{2} e^{q \nu_{1} \varsigma\left(\imath_{1}\right)}\left|\varsigma^{\prime}\left(\imath_{1}\right)\right|^{q} P_{\Pi_{m}, g_{1}}+m \Delta_{\varsigma}\left(q ; \nu_{1}, \nu_{2}, \imath_{1}, \imath_{2}\right) S_{\Pi_{m}, g_{1}, g_{2}}+e^{q \nu_{2} \varsigma\left(\imath_{2}\right)}\left|\varsigma^{\prime}\left(\imath_{2}\right)\right|^{q} P_{\Pi_{m}, g_{2}}\right. \\
\left.-c m\left(\imath_{2}-\imath_{1}\right)^{2}\left(m\left|\varsigma^{\prime}\left(\imath_{1}\right)\right|^{q} U_{\Pi_{m}, g_{1}, g_{2}}+\left|\varsigma^{\prime}\left(\imath_{2}\right)\right|^{q} T_{\Pi_{m}, g_{1}, g_{2}}\right)\right]^{\frac{1}{q}}
\end{gathered}
$$

where

$$
S_{\Pi_{m}, g_{1}, g_{2}}:=\int_{0}^{1}\left|\Pi_{m}(t)-\Pi_{m}(1-t)\right| g_{1}(t) g_{2}(t) d t
$$




$$
\begin{gathered}
P_{\Pi_{m}, g_{i}}:=\int_{0}^{1}\left|\Pi_{m}(t)-\Pi_{m}(1-t)\right|\left[g_{i}(t)\right]^{2} d t, \quad \forall i=1,2, \\
U_{\Pi_{m}, g_{1}, g_{2}}:=\int_{0}^{1}\left|\Pi_{m}(t)-\Pi_{m}(1-t)\right|\left[g_{1}(t)\right]^{2} g_{2}(t) d t \\
T_{\Pi_{m}, g_{1}, g_{2}}:=\int_{0}^{1}\left|\Pi_{m}(t)-\Pi_{m}(1-t)\right| g_{1}(t)\left[g_{2}(t)\right]^{2} d t
\end{gathered}
$$

and $\Delta_{\varsigma}\left(q ; \nu_{1}, \nu_{1}, \imath_{1}, \imath_{2}\right), B_{\Pi_{m}}(1)$ are defined as in Theorem 2.16 .

Proof. From Lemma 2.14, strongly exponentially generalized $\left(m, \nu_{1}, \nu_{2}, g_{1}, g_{2}\right)$ with modulus $c$ of $\varsigma$, generalized $\left(m, g_{1}, g_{2}\right)$-preinvexity of $\left|\varsigma^{\prime}\right|^{q}$, the wellknown power mean inequality and properties of the modulus, we have

$$
\begin{aligned}
& \left|\Xi_{\varsigma, \Pi_{m}}\left(\imath_{1}, \imath_{2}\right)\right| \leq \frac{\mathcal{F}_{\rho, \lambda}^{\sigma}\left(\imath_{2}-m \imath_{1}\right)}{2} \\
& \times \int_{0}^{1}\left|\Pi_{m}(t)-\Pi_{m}(1-t)\right|\left|e^{\varsigma\left(m \imath_{1}+t \mathcal{F}_{\rho, \lambda}^{\sigma}\left(\imath_{2}-m \imath_{1}\right)\right)} \varsigma^{\prime}\left(m \imath_{1}+t \mathcal{F}_{\rho, \lambda}^{\sigma}\left(\imath_{2}-m \imath_{1}\right)\right)\right| d t \\
& \leq \frac{\mathcal{F}_{\rho, \lambda}^{\sigma}\left(\imath_{2}-m \imath_{1}\right)}{2 \Pi_{m}(1)}\left[B_{\Pi_{m}}(1)\right]^{1-\frac{1}{q}} \\
& \times\left(\int_{0}^{1}\left|\Pi_{m}(t)-\Pi_{m}(1-t)\right| e^{q \varsigma\left(m \imath_{1}+t \mathcal{F}_{\rho, \lambda}^{\sigma}\left(\imath_{2}-m \imath_{1}\right)\right)}\left|\varsigma^{\prime}\left(m \imath_{1}+t \mathcal{F}_{\rho, \lambda}^{\sigma}\left(\imath_{2}-m \imath_{1}\right)\right)\right|^{q} d t\right)^{\frac{1}{q}} \\
& \leq \frac{\mathcal{F}_{\rho, \lambda}^{\sigma}\left(\imath_{2}-m \imath_{1}\right)}{2 \Pi_{m}(1)}\left[B_{\Pi_{m}}(1)\right]^{1-\frac{1}{q}} \\
& \times\left\{\int_{0}^{1}\left|\Pi_{m}(t)-\Pi_{m}(1-t)\right|\left[m g_{1}(t) e^{q \nu_{1} \varsigma\left(\imath_{1}\right)}+g_{2}(t) e^{q \nu_{2} \varsigma\left(\imath_{2}\right)}-c m\left(\imath_{2}-\imath_{1}\right)^{2} g_{1}(t) g_{2}(t)\right]\right. \\
& \left.\times\left[m g_{1}(t)\left|\varsigma^{\prime}\left(\imath_{1}\right)\right|^{q}+g_{2}(t)\left|\varsigma^{\prime}\left(\imath_{2}\right)\right|^{q}\right] d t\right\}^{\frac{1}{q}} \\
& =\frac{\mathcal{F}_{\rho, \lambda}^{\sigma}\left(\imath_{2}-m \imath_{1}\right)}{2 \Pi_{m}(1)}\left[B_{\Pi_{m}}(1)\right]^{1-\frac{1}{q}} \\
& \times\left[m^{2} e^{q \nu_{1} \varsigma\left(\imath_{1}\right)}\left|\varsigma^{\prime}\left(\imath_{1}\right)\right|^{q} P_{\Pi_{m}, g_{1}}+m \Delta_{\varsigma}\left(q ; \nu_{1}, \nu_{2}, \imath_{1}, \imath_{2}\right) S_{\Pi_{m}, g_{1}, g_{2}}+e^{q \nu_{2} \varsigma\left(\imath_{2}\right)}\left|\varsigma^{\prime}\left(\imath_{2}\right)\right|^{q} P_{\Pi_{m}, g_{2}}\right.
\end{aligned}
$$




$$
\left.-c m\left(\imath_{2}-\imath_{1}\right)^{2}\left(m\left|\varsigma^{\prime}\left(\imath_{1}\right)\right|^{q} U_{\Pi_{m}, g_{1}, g_{2}}+\left|\varsigma^{\prime}\left(\imath_{2}\right)\right|^{q} T_{\Pi_{m}, g_{1}, g_{2}}\right)\right]^{\frac{1}{q}} .
$$

The proof of Theorem 2.20 is completed.

We point out some special cases of Theorem 2.20.

Corollary 2.21. In Theorem 2.20 , taking $c \rightarrow 0^{+}$, we get

$$
\left|\Xi_{\varsigma, \Pi_{m}}\left(\imath_{1}, \imath_{2}\right)\right| \leq \frac{\mathcal{F}_{\rho, \lambda}^{\sigma}\left(\imath_{2}-m \imath_{1}\right)}{2 \Pi_{m}(1)}\left[B_{\Pi_{m}}(1)\right]^{1-\frac{1}{q}}
$$

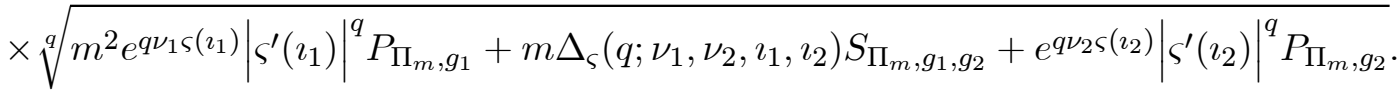

Corollary 2.22. In Theorem 2.20 , if we choose $\phi(t)=t$, we have

$$
\begin{aligned}
& \left|\frac{e^{\varsigma\left(m \imath_{1}\right)}+e^{\varsigma\left(m \imath_{1}+\mathcal{F}_{\rho, \lambda}^{\sigma}\left(\imath_{2}-m \imath_{1}\right)\right)}}{2}-\frac{1}{\mathcal{F}_{\rho, \lambda}^{\sigma}\left(\imath_{2}-m \imath_{1}\right)} \int_{m \imath_{1}}^{m \imath_{1}+\mathcal{F}_{\rho, \lambda}^{\sigma}\left(\imath_{2}-m \imath_{1}\right)} e^{\varsigma(t)} d t\right| \\
& \leq 2^{\frac{1-2 q}{q}} \mathcal{F}_{\rho, \lambda}^{\sigma}\left(\imath_{2}-m \imath_{1}\right) \\
& \times\left\{m^{2} e^{q \nu_{1} \varsigma\left(\imath_{1}\right)}\left|\varsigma^{\prime}\left(\imath_{1}\right)\right|^{q} \Omega_{g_{1}}+m \Delta_{\varsigma}\left(q ; \nu_{1}, \nu_{2}, \imath_{1}, \imath_{2}\right) \Theta_{g_{1}, g_{2}}+e^{q \nu_{2} \varsigma\left(\imath_{2}\right)}\left|\varsigma^{\prime}\left(\imath_{2}\right)\right|^{q} \Omega_{g_{2}}\right. \\
& \left.-c m\left(\imath_{2}-\imath_{1}\right)^{2}\left(m\left|\varsigma^{\prime}\left(\imath_{1}\right)\right|^{q} Q_{g_{1}, g_{2}}+\left|\varsigma^{\prime}\left(\imath_{2}\right)\right|^{q} R_{g_{1}, g_{2}}\right)\right\}^{\frac{1}{q}},
\end{aligned}
$$

where

(2.38) $\Theta_{g_{1}, g_{2}}:=\int_{0}^{1}|2 t-1| g_{1}(t) g_{2}(t) d t, \quad \Omega_{g_{i}}:=\int_{0}^{1}|2 t-1|\left[g_{i}(t)\right]^{2} d t, \quad \forall i=1,2$,

$$
Q_{g_{1}, g_{2}}:=\int_{0}^{1}|2 t-1|\left[g_{1}(t)\right]^{2} g_{2}(t) d t, \quad R_{g_{1}, g_{2}}:=\int_{0}^{1}|2 t-1| g_{1}(t)\left[g_{2}(t)\right]^{2} d t
$$


Corollary 2.23. In Theorem 2.20, taking $c \rightarrow 0^{+}$, where $\phi(t)=\frac{t^{\alpha}}{\Gamma(\alpha)}$ for $\alpha>0$ and $\mathcal{F}_{\rho, \lambda}^{\sigma}\left(\imath_{2}-m \imath_{1}\right)=\imath_{2}-m \imath_{1}$, we obtain

$$
\begin{aligned}
\mid \frac{e^{\varsigma\left(m \imath_{1}\right)}+e^{\varsigma\left(\imath_{2}\right)}}{2}- & \frac{\Gamma(\alpha+1)}{2\left(\imath_{2}-m \imath_{1}\right)^{\alpha}} \times\left\{J_{\left(m \imath_{1}\right)^{+}}^{\alpha} e^{\varsigma\left(\imath_{2}\right)}+J_{\imath_{2}^{-}}^{\alpha} e^{\varsigma\left(m \imath_{1}\right)}\right\} \mid \\
\leq & \frac{\left(\imath_{2}-m \imath_{1}\right)}{2}\left[B_{\Pi_{m}}(1)\right]^{1-\frac{1}{q}}
\end{aligned}
$$

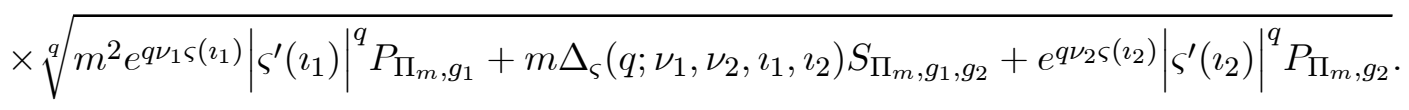

Remark 2.24. Under the conditions of Theorems 2.16 and 2.20, using remark 2.4, for the appropriate choices of function $\phi(t)=t, \frac{t^{\frac{\alpha}{k}}}{k \Gamma_{k}(\alpha)} ; \phi(t)$ $=\frac{t}{\alpha} \exp \left[\left(-\frac{1-\alpha}{\alpha}\right) t\right]$ where $\alpha \in(0,1)$, etc., we can get several new integral inequalities. Also, under assumptions of Theorems 2.16 and 2.20, taking $K=\left\|\varsigma^{\prime}\right\|_{\infty}:=\sup _{x \in O}\left|\varsigma^{\prime}(x)\right|$, we can deduce many other inequalities.

\section{Applications}

In this section, we provide some new error estimates for trapezoidal quadrature formula. Let $P$ be the partition of the points $\imath_{1}=x_{0}<x_{1}<\ldots<$ $x_{k}=\imath_{2}$ of the interval $\left[\imath_{1}, \imath_{2}\right]$. Let consider the following quadrature formula:

$$
\int_{\imath_{1}}^{\imath_{2}} e^{\varsigma(x)} d x=T(\varsigma, P)+E(\varsigma, P)
$$

where

$$
T(\varsigma, P)=\sum_{i=0}^{k-1}\left[\frac{e^{\varsigma\left(x_{i}\right)}+e^{\varsigma\left(x_{i+1}\right)}}{2}\right]\left(x_{i+1}-x_{i}\right)
$$

is the trapezoidal version and $E(\varsigma, P)$ is denote the associated approximation error.

Proposition 3.1. Let $\varsigma:\left[\imath_{1}, \imath_{2}\right] \rightarrow(0,+\infty)$ be a differentiable strongly exponentially generalized $\left(\nu_{1}, \nu_{2}, g_{1}, g_{2}\right)$-convex function with modulus $c>$ 0 on $\left(\imath_{1}, \imath_{2}\right)$, where $\imath_{1}<\imath_{2}$ and $\nu_{1}, \nu_{2} \in \mathbf{R}$. Suppose $g_{1}, g_{2}:[0,1] \rightarrow[0,+\infty)$ 
be continuous functions. If $\left|\varsigma^{\prime}\right|^{q}$ is generalized $\left(g_{1}, g_{2}\right)$-convex on $\left[\imath_{1}, \imath_{2}\right]$ for $q>1$ and $\frac{1}{p}+\frac{1}{q}=1$, then

$$
|E(\varsigma, P)| \leq \frac{1}{2 \sqrt[p]{p+1}} \times \sum_{i=0}^{k-1}\left(x_{i+1}-x_{i}\right)^{2}
$$

$$
\begin{gathered}
\times\left[e^{q \nu_{1} \varsigma\left(x_{i}\right)}\left|\varsigma^{\prime}\left(x_{i}\right)\right|^{q} G_{g_{1}}+\Delta_{\varsigma}\left(q ; \nu_{1}, \nu_{2}, x_{i}, x_{i+1}\right) F_{g_{1}, g_{2}}+e^{q \nu_{2} \varsigma\left(x_{i+1}\right)}\left|\varsigma^{\prime}\left(x_{i+1}\right)\right|^{q} G_{g_{2}}\right. \\
\left.-c\left(x_{i+1}-x_{i}\right)^{2}\left(\left|\varsigma^{\prime}\left(x_{i}\right)\right|^{q} M_{g_{1}, g_{2}}+\left|\varsigma^{\prime}\left(x_{i+1}\right)\right|^{q} N_{g_{1}, g_{2}}\right)\right]^{\frac{1}{q}}
\end{gathered}
$$

where

$$
\Delta_{\varsigma}\left(q ; \nu_{1}, \nu_{2}, x_{i}, x_{i+1}\right):=e^{q \nu_{1} \varsigma\left(x_{i}\right)}\left|\varsigma^{\prime}\left(x_{i+1}\right)\right|^{q}+e^{q \nu_{2} \varsigma\left(x_{i+1}\right)}\left|\varsigma^{\prime}\left(x_{i}\right)\right|^{q}
$$

and $F_{g_{1}, g_{2}}, G_{g_{1}}, G_{g_{2}}, M_{g_{1}, g_{2}}, N_{g_{1}, g_{2}}$ are defined as in Theorem 2.16.

Proof. Applying Theorem 2.16 for $m=1, \mathcal{F}_{\rho, \lambda}^{\sigma}\left(\imath_{2}-m \imath_{1}\right)=\imath_{2}-m \imath_{1}$ and $\phi(t)=t$ on the subintervals $\left[x_{i}, x_{i+1}\right](i=0, \ldots, k-1)$ of the partition $P$, we have

$$
\left|\frac{e^{\varsigma\left(x_{i}\right)}+e^{\varsigma\left(x_{i+1}\right)}}{2}-\frac{1}{x_{i+1}-x_{i}} \int_{x_{i}}^{x_{i+1}} e^{\varsigma(x)} d x\right| \leq \frac{\left(x_{i+1}-x_{i}\right)}{2 \sqrt[p]{p+1}}
$$

$$
\begin{gathered}
\times\left[e^{q \nu_{1} \varsigma\left(x_{i}\right)}\left|\varsigma^{\prime}\left(x_{i}\right)\right|^{q} G_{g_{1}}+\Delta_{\varsigma}\left(q ; \nu_{1}, \nu_{2}, x_{i}, x_{i+1}\right) F_{g_{1}, g_{2}}+e^{q \nu_{2} \varsigma\left(x_{i+1}\right)}\left|\varsigma^{\prime}\left(x_{i+1}\right)\right|^{q} G_{g_{2}}\right. \\
\left.-c\left(x_{i+1}-x_{i}\right)^{2}\left(\left|\varsigma^{\prime}\left(x_{i}\right)\right|^{q} M_{g_{1}, g_{2}}+\left|\varsigma^{\prime}\left(x_{i+1}\right)\right|^{q} N_{g_{1}, g_{2}}\right)\right]^{\frac{1}{q}} .
\end{gathered}
$$

Hence from (3.3), we get

$$
\begin{gathered}
|E(\varsigma, P)|=\left|\int_{a}^{b} e^{\varsigma(x)} d x-T(\varsigma, P)\right| \\
\leq\left|\sum_{i=0}^{k-1}\left\{\int_{x_{i}}^{x_{i+1}} e^{\varsigma(x)} d x-\frac{e^{\varsigma\left(x_{i}\right)}+e^{\varsigma\left(x_{i+1}\right)}}{2}\left(x_{i+1}-x_{i}\right)\right\}\right|
\end{gathered}
$$




$$
\begin{gathered}
\leq \sum_{i=0}^{k-1}\left|\left\{\int_{x_{i}}^{x_{i+1}} e^{\varsigma(x)} d x-\frac{e^{\varsigma\left(x_{i}\right)}+e^{\varsigma\left(x_{i+1}\right)}}{2}\left(x_{i+1}-x_{i}\right)\right\}\right| \\
\leq \frac{1}{2 \sqrt[p]{p+1}} \times \sum_{i=0}^{k-1}\left(x_{i+1}-x_{i}\right)^{2} \\
\times\left[e^{q \nu_{1} \varsigma\left(x_{i}\right)}\left|\varsigma^{\prime}\left(x_{i}\right)\right|^{q} G_{g_{1}}+\Delta_{\varsigma}\left(q ; \nu_{1}, \nu_{2}, x_{i}, x_{i+1}\right) F_{g_{1}, g_{2}}+e^{q \nu_{2} \varsigma\left(x_{i+1}\right)}\left|\varsigma^{\prime}\left(x_{i+1}\right)\right|^{q} G_{g_{2}}\right. \\
\left.-c\left(x_{i+1}-x_{i}\right)^{2}\left(\left|\varsigma^{\prime}\left(x_{i}\right)\right|^{q} M_{g_{1}, g_{2}}+\left|\varsigma^{\prime}\left(x_{i+1}\right)\right|^{q} N_{g_{1}, g_{2}}\right)\right]^{\frac{1}{q}} .
\end{gathered}
$$

The proof of Proposition 3.1 is completed.

Proposition 3.2. Let $\varsigma:\left[\imath_{1}, \imath_{2}\right] \rightarrow(0,+\infty)$ be a differentiable strongly exponentially generalized $\left(\nu_{1}, \nu_{2}, g_{1}, g_{2}\right)$-convex function with modulus $c>$ 0 on $\left(\imath_{1}, \imath_{2}\right)$, where $\imath_{1}<\imath_{2}$ and $\nu_{1}, \nu_{2} \in \mathbf{R}$. Suppose $g_{1}, g_{2}:[0,1] \rightarrow[0,+\infty)$ be continuous functions. If $\left|\varsigma^{\prime}\right|^{q}$ is generalized $\left(g_{1}, g_{2}\right)$-convex on $\left[\imath_{1}, \imath_{2}\right]$ for $q \geq 1$, then

$$
|E(\varsigma, P)| \leq 2^{\frac{1-2 q}{q}} \times \sum_{i=0}^{k-1}\left(x_{i+1}-x_{i}\right)^{2}
$$

$$
\begin{gathered}
\times\left[e^{q \nu_{1} \varsigma\left(x_{i}\right)}\left|\varsigma^{\prime}\left(x_{i}\right)\right|^{q} \Omega_{g_{1}}+\Delta_{\varsigma}\left(q ; \nu_{1}, \nu_{2}, x_{i}, x_{i+1}\right) \Theta_{g_{1}, g_{2}}+e^{q \nu_{2} \varsigma\left(x_{i+1}\right)}\left|v^{\prime}\left(x_{i+1}\right)\right|^{q} \Omega_{g_{2}}\right. \\
\left.-c\left(x_{i+1}-x_{i}\right)^{2}\left(\left|\varsigma^{\prime}\left(x_{i}\right)\right|^{q} Q_{g_{1}, g_{2}}+\left|\varsigma^{\prime}\left(x_{i+1}\right)\right|^{q} R_{g_{1}, g_{2}}\right)\right]^{\frac{1}{q}}
\end{gathered}
$$

where $\Delta_{\varsigma}\left(q ; \nu_{1}, \nu_{2}, x_{i}, x_{i+1}\right)$ is defined from (3.2), $\Theta_{g_{1}, g_{2}}, \Omega_{g_{1}}, \Omega_{g_{2}} ; Q_{g_{1}, g_{2}}, R_{g_{1}, g_{2}}$ are defined respectively from (2.38) and (2.39).

Proof. The proof is analogous as to that of Proposition 3.1, taking $m=1, \mathcal{F}_{\rho, \lambda}^{\sigma}\left(\imath_{2}-m \imath_{1}\right)=\imath_{2}-m \imath_{1}$ and $\phi(t)=t$ in Theorem 2.20.

Remark 3.3. Under the conditions of Theorems 2.16 and 2.20, using remark 2.4, for the appropriate choices of function $\phi(t)=t, \frac{t^{\frac{\alpha}{k}}}{k \Gamma_{k}(\alpha)} ; \phi(t)$ $=\frac{t}{\alpha} \exp \left[\left(-\frac{1-\alpha}{\alpha}\right) t\right]$ where $\alpha \in(0,1)$, etc., we can deduce several new bounds for the trapezoidal quadrature formula using above ideas and techniques. Also, under assumptions of Theorems 2.16 and 2.20, taking $K=$ $\left\|\varsigma^{\prime}\right\|_{\infty}:=\sup _{x \in O}\left|\varsigma^{\prime}(x)\right|$, we can obtain many other new bounds. 


\section{Conclusion}

The so-called strongly exponentially generalized $\left(m, \nu_{1}, \nu_{2}, g_{1}, g_{2}\right)$ functions can be applied to obtain several results in convex analysis, related optimization theory and may stimulate further research in different areas of pure and applied sciences.

\section{References}

[1] G. Alirezaei and R. Mathar, "On exponentially concave functions and their impact in information theory", 2018 Information Theory and Applications Workshop (ITA), San Diego, California, 2018. doi: 10.1109/ ita.2018.8503202

[2] T. Antczak, "( $p, r)$-invex sets and functions", Journal of mathematical analysis and applications, vol. 263, no. 2, pp. 355-379, 2001. doi: 10.1006/jmaa.2001.7574

[3] T. Antczak, "Mean value in invexity analysis", Nonlinear analysis: theory, methods and applications, vol. 60, no. 8, pp. 1473-1484, 2005. doi: 10.1016/j.na.2004.11.005

[4] S. M. Aslani, M. R. Delavar and S. M. Vaezpour, "Inequalities of fejer type related to generalized convex functions", International journal of analysis and applications, vol. 16, no. 1, pp. 38-49, 2018. doi: 10.28924/ 2291-8639-16-2018-38

[5] M. U. Awan, M. A. Noor, and K. I. Noor, "Hermite-Hadamard inequalities for exponentially convex functions", Applied mathematics and information sciences, vol. 12, no. 2, pp. 405-409, 2018. doi: 10.18576/ amis/ 120215

[6] F. X. Chen and S. H. Wu, "Several complementary inequalities to inequalities of Hermite-Hadamard type for S-convex functions", Journal of nonlinear sciences and applications, vol. 9, no. 2, pp. 705-716, 2016. doi: $10.22436 /$ jnsa.009.02.32

[7] Y.-M. Chu, M. A. Khan, T. U. Khan, and T. Ali, "Generalizations of hermite-hadamard type inequalities for MT-convex functions", Journal of nonlinear sciences and applications, vol. 09, no. 06, pp. 4305-4316, 2016. doi: 10.22436/jnsa.009.06.72

[8] M. Rostamian Delavar and M. De La Sen, "Some generalizations of hermite-hadamard type inequalities", SpringerPlus, vol. 5, no. 1, 2016. doi: 10.1186/ s40064-016-3301-3

[9] S. S. Dragomir, "On some new inequalities of Hermite-Hadamard type for $\mathrm{m}$-convex functions", Tamkang journal of mathematics, vol. 33, no. 1, pp. 45-56, 2002. doi: 10.5556/j.tkjm.33.2002.304 
[10] T. S. Du, M. U. Awan, A. Kashuri, and S. Zhao, "Some K-fractional extensions of the trapezium inequalities through generalized relative semi-(m,h)-preinvexity", Applicable analysis, vol. 100, no. 3, pp. 642-662, 2019. doi: 10.1080/ 00036811.2019.1616083

[11] T.-S. Du, J.-G. Liao, and Y.-J. Li, "Properties and integral inequalities of hadamard- Simpson type for the generalized (s, m) -preinvex functions", Journal of nonlinear sciences and applications, vol. 9, no. 5, pp. 3112-3126, 2016. doi: 10.22436/jnsa.009.05.102

[12] M. Jleli, "On Hermite-Hadamard type inequalities via fractional integrals of a function with respect to another function", Journal of nonlinear sciences and applications, vol. 9, no. 3, pp. 1252-1260, 2016. doi: 10.22436/jnsa.009.03.50

[13] J. Hristov, "Response functions in linear viscoelastic constitutive equations and related fractional operators", Mathematical modelling of natural phenomena, vol. 14 , no. 3, pp. 1-34, 2019. doi: 10.1051/ mmnp/ 2018067

[14] U. N. Katugampola, "New approach to a generalized fractional integral", Applied mathematics and computation, vol. 218, no. 3, pp. 860-865, 2011. doi: 10.1016/j.amc.2011.03.062

[15] A. Kashuri and R. Liko, "Some new Hermite-Hadamard type inequalities and their applications", Studia scientiarum mathematicarum hungarica, vol. 56, no. 1, pp. 103-142, 2019. doi: 10.1556/ 012.2019.56.1.1418

[16] T. Lara, N. Merentes, R. Quintero and E. Rosales, "On strongly m-convex functions", Mathematica aeterna, vol. 5, no. 3, pp. 521-535, 2015.

[17] T. Lara, N. Merentes, R. Quintero and E. Rosales, "On inequalities of Fejer and Hermite-Hadamard types for strongly m-convex functions", Mathematica aeterna, vol. 5, no. 5, pp. 777-793, 2015.

[18] W. Liu, W. Wen, and J. Park, "Hermite-Hadamard type inequalities for MT-convex functions via classical integrals and fractional integrals", Journal of nonlinear sciences and applications, vol. 9, no. 3, pp. 766-777, 2016. doi: 10.22436/jnsa.009.03.05

[19] C. Luo, T. S. Du, M. A. Khan, A. Kashuri and Y. Shen, "Some k-fractional integrals inequalities through generalized $\lambda_{\varphi \mathrm{m}}$-preinvexity", Journal of computational analysis and applications, vol. 27, no. 4, pp. 690-705, 2019 .

[20] M. Matłoka, "Inequalities for H-preinvex functions", Applied mathematics and computation, vol. 234, pp. 52-57, 2014. doi: 10.1016/j.amc. 2014.02.030

[21] M. V. Mihai, "Some Hermite-Hadamard type inequalities obtain via Riemann-Liouville fractional calculus", Tamkang journal of mathematics, vol. 44, no. 4, pp. 411-416, 2013. doi: 10.5556/ j.tkjm.44.2013.1218 
[22] K. S. Miller and B. Ross, An introduction to the fractional calculus and fractional differential equations, New York: Wiley, 1993.

[23] M. A. Noor, "Some new classes of non convex functions", Nonlinear functional analysis and applications, vol. 11, no. 1, pp. 165-171, 2006.

[24] M. A. Noor and K. I. Noor, "Strongly exponentially convex functions and their properties", Journal of advanced mathematical studies, vol. 12, no. 2, pp. 177-185, 2019. [On line]. Available: https:/ / bit.ly/ 3Kv8ue3

[25] M. A. Noor and K. I. Noor, "Strongly exponentially convex functions", UPB scientific bulletin, series a: applied mathematics and physics, vol. 81, no. 4, 2019. [On line]. Available: https:/ / bit.ly/ 3fNAXO4

[26] M. A. Noor and K. I. Noor, “Exponential convex functions”, Preprint.

[27] M. A. Noor and K. I. Noor and S. Rashid, "Fractal exponential convex functions and inequalities", Preprint.

[28] M. A. Noor and K. I. Noor and S. Rashid, "Exponential r-convex functions and inequalities", Preprint.

[29] O. Omotoyinbo and A. Mogbodemu, "Some new Hermite-Hadamard integral inequalities for convex functions", International journals of science and innovation technology, vol. 1, no. 1, pp. 1-12, 2014.

[30] M. E. Özdemir, S. S. Ragomir, and Ç. Yildiz, "The Hadamard inequality for convex function via fractional integrals", Acta mathematica scientia, vol. 33, no. 5, pp. 1293-1299, 2013. doi: 10.1016/ S0252-9602(13)60081-8

[31] J. Pečarić and J. Jakšetić, "Exponential convexity, Euler-Radau expansions and stolarsky means", Rad Hrvatske akademije znanosti i umjetnosti : Matematičke znanosti, vol. 515, pp. 81-94, 2013. [On line]. Available: https:/ / bit.ly/ 35dJEiE

[32] C. Peng, C. Zhou, C. and T. S. Du, “Riemann-Liouville fractional Simpson's inequalities through generalized $\left(\mathrm{m}, \mathrm{h}_{1}, \mathrm{~h}_{2}\right)$-preinvexity", Italian journal of pure and applied mathematic, vol. 38, pp. 345-367, 2017. [On line]. Available: https:/ / bit.ly/ 3Iu1Ym6

[33] R. K. Raina, "On generalized Wright's hypergeometric functions and fractional calculus operators", East asian mathematics journal, vol. 21, no. 2, pp. 191-203, 2005. [On line]. Available: https:/ / bit.ly/ 3AiV1kQ 
[34] S. Rashid, M. A. Noor, and K. I. Noor, "Fractional exponentially m- convex functions and inequalities", International Journal of Analysis and Applications, vol. 17, no. 3, pp. 464-478, 2019.

[35] M. A. F. dos Santos, "Fractional Prabhakar derivative in diffusion equation with non-static stochastic resetting", Physics, vol. 1, no. 1, pp. 40-58, 2019. doi: 10.3390/ physics 1010005

[36] M. Z. Sarikaya and F. Ertuğral, "On the generalized Hermite- Hadamard inequalities", Annals of the University of Craiova, Mathematics and Computer Science Series, vol. 47, no. 1, pp. 193-213. [On line]. Available: https:/ / bit.ly/ 3Ax1Dfs

[37] M. Z. Sarikaya and H. Yildirim, "On generalization of the Riesz potential", Indian journal of mathematical sciences, vol. 3, no. 2, pp. 231-235, 2007.

[38] H. Wang, T. S. Du and Y. Zhang, "k-fractional integral trapezium- like inequalities through ( $h, \mathrm{~m})$-convex and $(\alpha, \mathrm{m})$-convex mappings", Journal of Inequalities and Applications, vol. 2017, Art. ID. 311, 2017. doi: 10.1186/ s13660-017-1586-6

[39] Y. Zhang, T. S. Du, H. Wang, Y. J. Shen, and A. Kashuri, "Extensions of different type parameterized inequalities for generalized $(\mathrm{m}, \mathrm{h})$ preinvex mappings via k-fractional integrals", Journal of inequalities and applications, vol. 2018, Art. ID. 49, 2018. doi:10.1186/ s13660-018-1639-5 


\section{Artion Kashuri}

Department of Mathematics,

Faculty of Technical Science,

University Ismail Qemali,

Vlora,

Albania

e-mail: artionkashuri@gmail.com

Corresponding author

and

\section{Rozana Liko}

Department of Mathematics, Faculty of Technical Science, University Ismail Qemali, Vlora,

Albania

e-mail: rozanaliko86@gmail.com 